\title{
Human-computer interaction for users with cerebral palsy based on head orientation. \\ Can cursor's movement Be modeled by Fitts's law?
}

\author{
Miguel A. Velasco ${ }^{\mathrm{a}, *}$, Alejandro Clemotte ${ }^{\mathrm{a}}$, Rafael Raya ${ }^{\mathrm{b}}$, Ramón Ceres $^{\mathrm{c}}$, Eduardo Rocon ${ }^{\mathrm{a}}$ \\ ${ }^{a}$ Neural and Cognitive Engineering Group, Centre for Automation and Robotics (CAR) CSIC-UPM, Ctra. Campo Real Km 0.2, 28500 Arganda \\ del Rey, Spain \\ ${ }^{b}$ Department of Information Technologies, Universidad CEU San Pablo, Urbanización Montepríncipe, 28668 Boadilla del Monte, Spain \\ ${ }^{c}$ Cajal Institute CSIC. Av. Doctor Arce, 37. 28002 Madrid, Spain
}

\begin{abstract}
This paper presents an experiment to validate a head-mounted inertial interface for human-computer interaction (HCI) developed for people with cerebral palsy (CP). The method is based on Fitts's law, an empirical model of human motor performance for aimed movements. Head motion is recorded in a series of goal-crossing tasks and a regression model of the movement time $(M T)$ is estimated for each user. Values of $R^{2}$ above 0.9 are indicators of a strong correlation of those motion patterns with the linear model proposed by Fitts. The analysis of $M T$ confirmed that head movements of users without disability follow Fitts's law and showed that 3 users with CP (MACS IV and V) had the same behavior. There was a weaker correlation $\left(R^{2}=0.839\right)$ for one individual with cervical dystonia and ballistic movements and no correlation for two users with cervical hypotonia and dyskinetic CP. Results show the impact of ballistic movements and poor postural control in computer interaction. They also provide the foundation for new interaction techniques to develop a universal computer interface for motor impaired users.
\end{abstract}

Keywords:

Fitts's law; Inertial sensor; Human-computer interaction; Cerebral palsy; Motor disorder; Head orientation

\section{Introduction}

Cerebral palsy $(\mathrm{CP})$ is one of the most severe disabilities in childhood and makes heavy demands on health, educational, and social services as well as on families and children themselves. Bax et al. (2005) defined CP as 'a disorder of movement and posture due to a defect or lesion of the immature brain'. Those disorders of posture and movement which are (1) of short duration, (2) due to progressive disease or (3) due solely to mental deficiency are usually excluded from CP. The prevalence of CP is internationally 2.2 cases per 1000 births (Van Naarden Braun et al. (2016)). The overall rate for the period from 1980 to 1990 was 2.08 per 1000 live births and there are nearly 17 million people with $\mathrm{CP}$ worldwide of all ethnicities and social status (Johnson (2002)).

\footnotetext{
${ }^{*}$ Corresponding author.

Email address: miguel. velasco@csic .es (Miguel A. Velasco)

URL: g-nec . com (Miguel A. Velasco)
}

The work 'Surveillance of cerebral palsy in Europe: a collaboration of cerebral palsy surveys and registers' presented a consensus that was reached on a definition of CP, description, and classification in terms of diagnosis, topography and function (severity). Cans (2007) divides the CP into three types: spastic, ataxic and dyskinetic. Spastic CP is the most common form (70-80\% of the individuals with $\mathrm{CP}$ are affected by spasticity) and is characterized by at least two of these signs: abnormal pattern of posture and/or movement, increased tone, and pathological reflexes. It may be either bilateral or unilateral. Ataxic $\mathrm{CP}(6 \%)$ is characterized by both abnormal pattern of posture and/or movement and loss of orderly muscular coordination; movements are performed with abnormal force, rhythm, and accuracy. Dyskinetic CP (6\%) is dominated by both abnormal pattern of posture and/or movement; and involuntary, uncontrolled, recurring, occasionally stereotyped movements (Cans (2007)).

CP can affect different parts of the body. Unilat- 
eral and bilateral $\mathrm{CP}$ affect one or two sides of the body, respectively. Diplegia refers to an impairment that affects only the lower limbs, and quadriplegia is associated with an impairment in both upper and lower limbs. Manual ability is an issue in at least two-thirds of children with $\mathrm{CP}$ and it affects activities such as eating, dressing, writing or playing. Eliasson et al. (2006) presented the Manual Ability Classification System (MACS) to measure those limitations with 5 levels: from "handles objects easily and successfully" (MACS level I) to "does not handle objects and has severely limited ability to perform even simple actions" (MACS V). Severe motor disorders in combination with sensory and cognitive alterations result in great difficulties for some individuals with $\mathrm{CP}$ to communicate and interact with their environment.

In the recent years, the use of interactive tools and other apps developed for personal computers (PC), tablets, and smart-phones is spreading. Therapists and caregivers use them to overcome some of the effects of these motor limitations and promote neural plasticity, especially during childhood. Unfortunately, there are still barriers for these individuals to access the computer with standard input devices. This limitations are related to poor manipulation skills.

Computer access is often depicted as an act of pointing to graphical elements on the screen (Balakrishnan (2004)). Any pointing task consists of as an initial phase of ballistic movements followed by a slower homing phase. People with $\mathrm{CP}$ usually encounter navigation problems (difficulties moving the cursor during the ballistic phase), targeting errors (difficulties staying on a target in the homing phase), and other issues related to clicking (Hurst et al. (2008)).

Many authors have approached the development of alternative interfaces in response to the special needs of people with motor disorders. Those solutions are available in multiple devices (PC, tablets, smartphones) and allow different types of actions: pointing (Tuisku et al. (2014)), scanning (Biswas and Langdon (2013)) or scrolling (Zhao et al. (2014)). The interface translates voluntary actions, registered as physical gestures (Rempel et al. (2014)), muscle activity (Chen and Wang (2013)), gaze and or head tracking (Špakov et al. (2014)), voice, etc. or other measurements such as evoked potentials (Schlögl et al. (2009)) into input commands. Raya et al. (2012) proposed the ENLAZA interface, an adapted head control for users with severe motor disorders (especially CP) who cannot use traditional hand-held solutions to access the computer. ENLAZA employs the users' residual motor skills and is robust to their involuntary movements.
Davies et al. (2010a) reviewed over thirty devices and technologies which enabled or enhanced computer access for individuals with $\mathrm{CP}$, including pointing devices (Wu and Chen (2007)) and keyboard modifications (Lin et al. (2008)), adapted GUIs (Simpson et al. (2006)), filters and facilitation algorithms (Olds et al. (2008)) and speech and gesture recognition. While these solutions may enable computer access for this population, few of them had undergone systematic evaluation for CP. As Almanji et al. (2014) argued, the use of assistive technology for computer access encountered barriers that led to the use of typical mice, trackballs or touch screens for practical reasons. Fitts's law (Fitts (1954)) is one of the paradigms that the HCI community applies to assess usability. In this study, we aim to find the answer to the following questions:

1. Can we confirm that the ENLAZA pointing device, based on head motion, follows Fitts's law when it is used by people without motor disabilities?

2. If the answer to (1) is affirmative, does ENLAZA follow Fitts's law when it is used by people with CP? We will focus on those with diplegia and quadriplegia and manipulation skills classified as MACS IV and V.

To answer these questions, we will quantify how effectively users with and without $\mathrm{CP}$ are able to access the computer by modeling their movement times with Fitts's law. Even though Fitts's law describes, by definition, upper limb motor behavior, the works of Jagacinski and Monk (1985), Radwin et al. (1990), and Gump et al. (2002) suggest that the motion of the head during pointing tasks with a direct pointing device is of the same nature. Our first goal is to confirm that hypothesis on users without disabilities. Most of the work related to enabling computer access to individuals with motor disorders focuses in MACS levels I-III. The research directed to individuals with severe motor disorders (MACS IV and V) is scarce. Our second contribution is to assess the performance of the ENLAZA head mouse when it is used by individuals with CP MACS IV and V. While Davies et al. (2014) observed that Fitts's law cannot be applied in youths with CP, Almanji et al. (2014) specified that there was a correlation between speed and accuracy, but not as strong as in typically developing youths. We expect to find similar differences between users with and without CP in our study. The analysis of data will provide us with information about which profiles of motor impairment are modeled by Fitts's law in a heterogeneous group of users with CP. This new information can lead to changes in the calibration process or to the development of new control strategies for the 
interface based on head movement.

\section{Background}

\subsection{Pointing models}

Fitts's law is an empirical model which explains the trade-off characteristics between speed and accuracy for human movement during pointing tasks. The model was first developed for the optimization of worker efficiency during assembling tasks in production lines (Fitts (1954)). Since Card et al. (1978) proposed its application in Human-Computer Interaction (HCI), it has been widely used in the design and validation of graphical user interfaces (GUI).

Fitts's law models the human motor system as a communication channel with a certain bandwidth, measured in bits per second. Information is transmitted through the channel by performing a movement of a certain difficulty, measured in bits. Fitts defines a simple pointing task where the subject has to perform a movement of amplitude $A$ to reach a target of size $W$. The model states that movement time $(M T)$ is a linear function of the index of difficulty $(I D)$.

$$
M T=a+b * I D
$$

Parameters $a$ and $b$ are named intercept and slope. Soukoreff and Mackenzie (2004) suggest the use of the Shannon formulation, which provides a better fit with observations and is truer to the information theorem on which Fitts's law is based. It also prevents the estimation of negative values of $I D$. This formulation represents the $I D$ as:

$$
I D=\log _{2}\left(\frac{A}{W}+1\right)
$$

The standard ISO 9241-Part 9. Requirements for nonkeyboard input devices defines the Throughput, TP, to quantify the performance of a reaching task with pointing devices. It is based on the time needed to complete the task and the difficulty of the task and is considered a more robust parameter than the $M T$ itself. The difficulty of the task is quantified by the index of difficulty, $I D$, which is based on $A$ and $W$. The TP during a single task is:

$$
T P=\frac{I D}{M T}
$$

On the other hand, Wobbrock et al. (2011) extrapolated Fitts's model to a two-dimensional (2D) solution and compared two approaches for the estimation of the task Throughput: slope-inverse throughput, $T P_{i n v}$, and mean-of-means throughput, $T P_{a v g}$. They concluded that
$T P_{\text {avg }}$ agrees more across dimensionalities and exhibits smaller variance among users.

$$
\begin{gathered}
T P_{i n v}=\frac{1}{b} \\
T P_{a v g}=\frac{I D_{a v g}}{M T_{a v g}}
\end{gathered}
$$

Although each throughput calculation results in a bits/s measure, they consider different things. It is reasonable, then, to report all throughputs rather than adhere to one of them.

\subsection{Pointing strategies}

Wall and Harwin (2000) among others were critical of the classic reciprocal tapping because the subjects quickly learned to improve their performance during the repetitive action: which is "unrepresentative of most pointing tasks". The experiments assessing pointing devices and Fitts's law are generally designed following the standard proposed by Soukoreff and Mackenzie (2004). However, there are some scenarios and new ways of interaction that must be considered.

Quinn et al. (2013) simulated beginner and expert behavior with two pointing techniques (random task and bi-directional tapping). They described each trial as a 3-phase sequence (initiation, execution of movement and confirmation of cursor location) and found that the initiation phase in the random tasks was larger than in the bi-directional tapping. In a calibration stage random showed smaller correlation with the linear model than the bi-directional tapping that was perhaps due to the initiation phase component, although both of them were considered good matches $\left(R_{r n d}^{2}=0.96, R_{\text {tap }}^{2}=0.99\right)$.

Accot and Zhai (2002), Apitz and Guimbretière (2004), and Apitz et al. (2010) analyzed whether crossing boundaries could complement or replace the traditional enter object and select it paradigm with a stylus and observed that "goal-crossing indeed follows a quantitative relation among movement time, movement distance and the constraint of the goal width" and "that relationship takes the same form as in Fitts's law". Goalcrossing $M T$ s were shorter or no longer than pointing and selection under the same ID. Luo and Vogel (2014) extrapolated this analysis to direct touch input and confirmed Accot's hypothesis.

\subsection{Pointing devices}

Fitts's law has been often used to evaluate not only the mouse but also new input devices, mostly with nonimpaired users, with various results. Felton et al. (2012) 
and Kim et al. (2013) applied it to a brain-computer interface (BCI); Kim et al. (2015), to a BCI-gaze-tracking hybrid interface. A linear relationship between $M T$ and $I D$ was found for both pure and hybrid BCI interfaces.

Other interfaces showed different behavior due to the nature of the interaction. Tuisku et al. (2012) and Alonso et al. (2013) evaluated gaze-tracking interfaces with Fitts's law and found a poor correlation with the model, which is in contradiction with Surakka et al. (2004) and others. Alonso pointed out that MT depended on the size of the target more than on the amplitude of the movement as eye movements are extremely fast. Scheme et al. (2013) and Kamavuako et al. (2014) described two interfaces based on superficial and intramuscular EMG to move the computer cursor in one and two dimensions, respectively. Fitts's evaluation showed that $M T$ and $I D$ had a strong correlation when the reaching tasks involved movements in one axis, and was even stronger with intramuscular EMG. Scheme and Englehart (2013) and Williams and Kirsch (2015) assessed 3D motion with superficial EMG but Fitts's law could not be applied as participants tend to use sequential command strategies for combined movements in different axis for a more natural interaction.

Jagacinski and Monk (1985) and Rudigkeit et al. (2015) tested the applicability of Fitts's law for headcontrolled interfaces similar to the one that we evaluated in this study. Jagacinski and Monk even calculated three regression models. The results of both studies suggested that Fitts's law is suitable for head motion and non-impaired users. Radwin et al. (1990), Gump et al. (2002), and Raya et al. (2012) hypothesized the linear model could be also used with individuals with $\mathrm{CP}$ and obtained promising results. Unfortunately, the small number of participants in their studies (one, three, and two subjects) reduced the significance of their findings.

\subsection{The ENLAZA interface}

ENLAZA (Raya et al. (2012)) allows users to control the cursor of the computer with movements of their heads. It consists of a headset with a cap and an inertial measurement unit, IMU (Werium S.L., Spain) as depicted in Figs. 2(a) and 2(b). It integrates a tri-axial gyroscope, accelerometer, and magnetometer. It uses Coriolis force principle to measure angular velocity and Hooke's law for acceleration. The magnetometer measures Earth's magnetic field. The IMU design is based on MEMS technology and is available in a small package $(27 \times 35 \times 13 \mathrm{~mm}, 27$ grams). It is able to measure $+/-2.0$ Gauss, $+/-3 \mathrm{~g}$ and $+/-500^{\circ} / \mathrm{s}$ in the three axes.

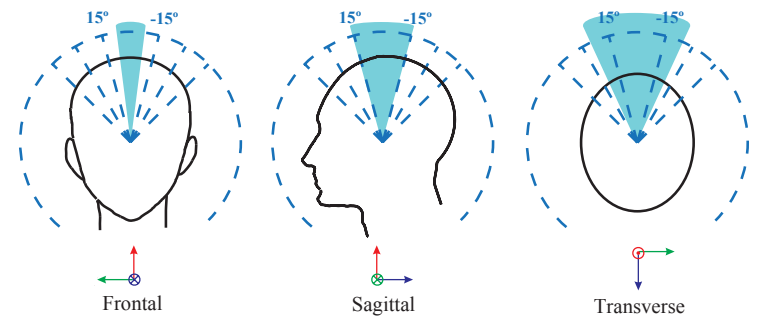

(a) User without motor disabilities.

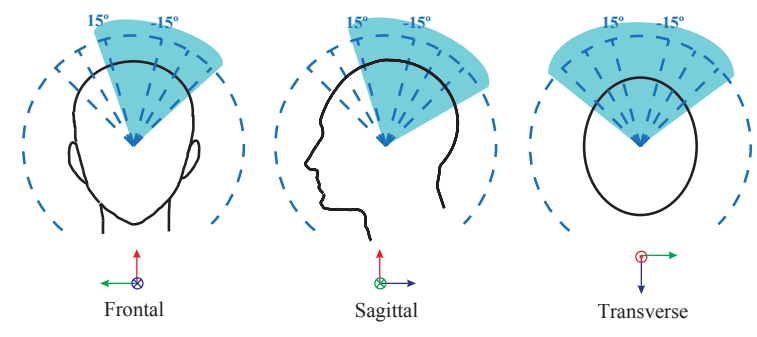

(b) User with cerebral palsy.

Figure 1: Representation of the head orientation in the frontal, sagittal and transverse planes. The Euler angles displayed are, from left to right, $\alpha, \beta$ and $\gamma$. Recordings correspond to a total of 16 reaching tasks with the ENLAZA interface.

The angular resolution of the device is $0.05^{\circ}$, a static accuracy less than one degree and a dynamic accuracy of about $2^{\circ}$ RMS.

IMU orientation is estimated based on the data recorded by the accelerometer, gyroscope, and magnetometer. The three Euler angles $\alpha, \beta$ and $\gamma$ (in the frontal, sagittal and transverse planes) are calculated from the rotation matrix:

$$
\begin{aligned}
& \boldsymbol{R}_{\boldsymbol{G S}}=\boldsymbol{R}_{\boldsymbol{S}} \cdot\left(\boldsymbol{R}_{\boldsymbol{G}}\right)^{-1} \\
& \alpha=\operatorname{atan}\left(-\frac{\boldsymbol{R}_{G S(2,3)}}{\boldsymbol{R}_{G S(3,3)}}\right) \\
& \beta=\operatorname{asin}\left(\boldsymbol{R}_{\boldsymbol{G S}(\mathbf{1 , 3})}\right) \\
& \gamma=\operatorname{atan}\left(-\frac{\boldsymbol{R}_{G S(\mathbf{1}, 2)}}{\boldsymbol{R}_{G S(1, \mathbf{1})}}\right)
\end{aligned}
$$

where $\boldsymbol{R}_{\boldsymbol{G}}$ is defined as the rotation matrix of the global reference system corresponding to the neutral position of the head (looking at the center of the screen) and $\boldsymbol{R}_{\boldsymbol{S}}$ as the rotation matrix that describes the orientation of the sensor at each frame. For the purpose of this study, the mouse pointer is controlled with an absolute control, meaning that there is a unique mapping between the head orientation and the location of the pointer. During a calibration process, a therapist adjusts the gain of the transfer function that translates the changes in the orientation of the head into a displacement of the pointer on 
the screen. After this process, all pixels in the screen are reachable for the user's head Range of Motion, ROM, described by Bible et al. (2010). Examples of different $R O M$ can be observed in Figs. 1(a) and 1(b).

During the development phase, many cases of overshoots and undershoots were found in users with preserved gross motor control but poor fine motor control. This caused a number of sub-movements around any target they tried to select. Raya et al. (2012) developed a Robust Kalman Filter (RKF) that facilitates fine motor control based on the characterization of involuntary movements found in users with cerebral palsy. The filter prevents the trajectory of the pointer from being affected by ballistic, athetoid, dystonic or other associated involuntary movements. An initial movement which rapidly covers distance is smoothly filtered, whereas the movements around the target are more attenuated. Experiments with 3 participants with CP showed drastic reductions (up to 65\%) in the number of sub-movements around the target.

Prior to this study, Raya et al. (2013) and Velasco et al. (2016) had used the inertial interface ENLAZA for the assessment of impairment. Two metrics were proposed: the frequency of movement and the ROM of user's head. $R O M$ is defined as the difference between the maximum and minimum Euler angles measured by the IMU in one of the anatomical planes: frontal, sagittal or transverse (Euler angles $\alpha, \beta$ and $\gamma)$. Results showed significant differences in the measured $R O M$ between healthy subjects and users with $\mathrm{CP}$ due to the motor control and posture disorders. Figs. 1(a) and 1(b) depict the three angles measured in one user of each study group. Head motion was analyzed in the frequency domain but no significant differences were found. This indicates that there is and overlap between the frequency components of the involuntary movements and with those of the voluntary motion in CP.

\section{Experiment}

We based our experiment on goal-crossing random tasks instead of the standard bi-directional tapping and selection proposed by Soukoreff and Mackenzie (2004). While this may seem to be rather unorthodox, we believe that the motor and cognitive profile of our participants with CP required a different approach. The standard task would be unattractive and tedious for most of our users and we are confident that the works of Accot and Zhai (2002), Quinn et al. (2013) and others support our experiment. Users wore the ENLAZA device to control the cursor of the computer with movements

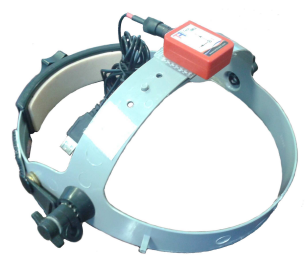

(a) IMU attached to the helmet.

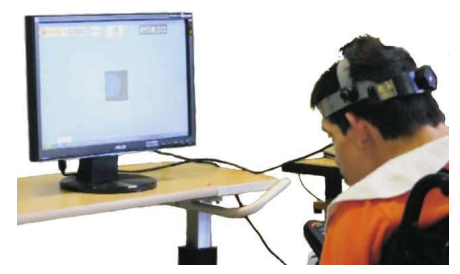

(b) Participant from the CP group.

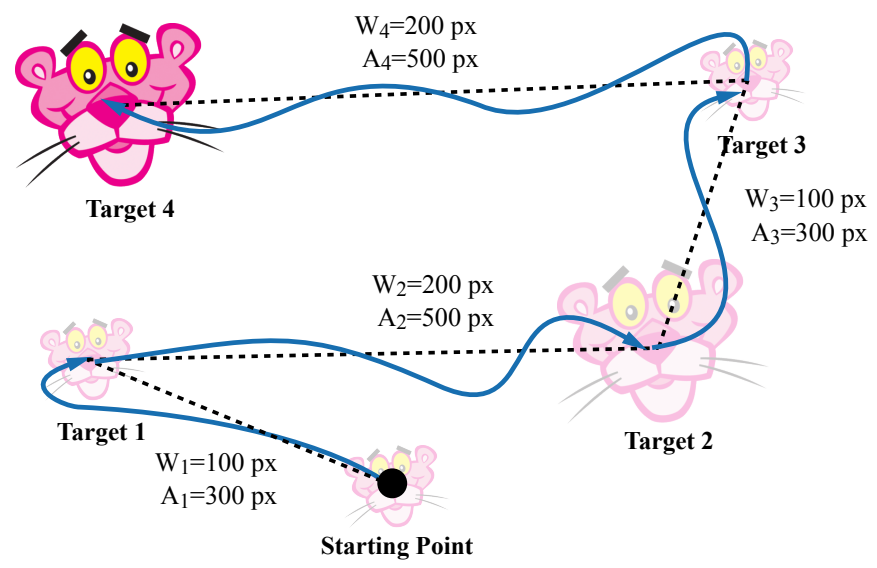

(c) Sequence of 4 random tasks with a goal-crossing strategy.

Figure 2: Experimental set up: the ENLAZA interface and representation of 4 random goal-crossing tasks in the Pink Panther videogame.

of their heads. They were instructed to locate the mouse cursor over a static target as quickly as possible. All the participants had previous experience with the device.

\subsection{Participants}

A total of 16 users were recruited. Six users without disabilities, ND group, (age 26.1+/-4.2) participated in the early experiments and completed $3+/-1$ training sessions before starting the study. Ten users with CP (age 31.8+/-9.2) started a second phase of the study at ASPACE-Cantabria (Santander, Spain), a center specialized in CP and similar disorders. This study was approved by the Ethics Committee of ASPACE-Cantabria and was in accordance with the Declaration of Helsinki on human research. Prior to the beginning of the tests, they had completed $21+/-7$ sessions with ENLAZA in 
Table 2: User description: relevant characteristics.

\begin{tabular}{ccccc}
\hline User & Cervical Tone & General Tone & Associated Movements & Intellectual ability \\
\hline CP1 & Dystonia & Dystonia & Ballistic movements & Normal \\
CP2 & Hypertonia & Hypertonia & Athetoid movements & Normal \\
CP3 & Hypotonia & Hypertonia & Dystonic movements & Normal \\
CP4 & Hypotonia & Hypotonia & No movements associated & Mild disability \\
CP5 & Hypertonia & Hypertonia & Athetoid movements & Medium disability \\
CP6 & Hypotonia & Hypotonia & No movements associated & Medium disability \\
\hline
\end{tabular}

two months and training games.

Table 1 depicts the classification of the participants in terms of diagnosis and topology of the motor disorder. It also depicts their manipulation skills. Some other descriptors that the therapists considered relevant for the study can be observed in Table 2. Deficits in trunk control affect head stability as pointed out by Saavedra et al. (2010) and Saavedra and Woollacott (2015). Consequently, pelvic and torso support was provided for those participants with poor trunk postural control.

Four of the participants with CP left the study after a small number of sessions. One of the participants left ASPACE-Cantabria. It was a personal decision that could not be prevented. Another two participants had poorer head control than the rest of the participants and could not complete some of their work sessions in the time assigned in each work session. Unfortunately, the schedules in ASPACE-Cantabria are tight and other activities such as PT or occupational therapy were the priority. Both continued using ENLAZA in less challenging activities. The fourth dropout was due to the fact that the participant did not actually meet the inclusion criteria. Although the therapists in ASPACE-Cantabria thought his cognitive development was not an obstacle for his performance, they soon realized that he did not fully understand the exercise. The tests from these participants are not included in the analysis.

\subsection{Goal-crossing random task}

A simple videogame (see Fig. 2) was developed using Visual C\# for the framework .NET 4.0. Each work session with the videogame consisted of reaching 17 targets on the screen: one for practicing (and to set the exercise's starting point) and 16 for assessment. The participants were asked to locate the cursor over a target on the screen "as fast as they could". The target was a Pink Panther's face inside a squared, transparent frame and there was high contrast between the pink target and the white background. Between 2 consecutive goal-crossing tasks, a brief scene corresponding to $1 / 17$ the length of a video was played in order to give the participants both a reward and time to rest. A new target appeared on the screen in a randomized order when the video scene stopped. The user would reach the end of the video after completing the $17^{\text {th }}$ task. No errors could be made as there was just one element on the screen at every time and there was no preset timeout.

The location of any new target was calculated as a function of the location of the cursor the very moment the video stopped. Two values of movement amplitude, $A$, were chosen; the target could be located at a distance of either 300 or 500 pixels from the position of the cursor. Similarly, two values of target size, $W$, were used: 100 or 200 pixels. Hence, there were 4 combinations of amplitude and width that provided us with values of $I D$ in a range of interest (see Section 3.3). In a session, the user had to perform four repetitions in a randomized order of those four $(A, W)$ combinations, for a total of 16 tasks. Fig. 2(c) shows a series of 4 goal-crossing tasks in a randomized order: there are targets of two different widths, $W$, located at two possible values of amplitude, $A$. The dashed line represents the ideal trajectory while the solid line represents the actual path taken by the user. In this case, the pairs $(A, W)$ are: $A_{1}=100$ px, $W_{1}=300 \mathrm{px} ; A_{2}=500 \mathrm{px}, W_{2}=200 \mathrm{px} ; A_{3}=300 \mathrm{px}$, $W_{3}=100 \mathrm{px} ; A_{4}=500 \mathrm{px}, W_{4}=200 \mathrm{px}$. Note that the first target (marked with the black dot) is used as the exercise's starting point.

The session was designed not to last more than 5 minutes in total, including goal-crossing task and the reproduction of the video. The therapists aimed to schedule two consecutive sessions for each participant and day. Between sessions, the participant could rest for five minutes. The average duration of each session was $3.76 \pm 0.67 \mathrm{~min}$ and the participants completed $1.8 \pm 0.67$ sessions per day of work. 


\subsection{Assessment of correlation with Fitts's law}

According to Fitts's law, the cursor's time of flight during a reaching task should be a logarithmic function of the target size and the amplitude of movement, thus a linear function of the $I D$. A set of tests was defined to measure the Movement Time (MT) during several goalcrossing tasks for predefined Indexes of Difficulty (ID). Subsequently, we estimated the $R^{2}$ value of the linear approximation of the MT-ID curves. As we mentioned earlier in the text, the tests are based on a series of tasks with 4 values of $I D: 1.32,1.8,2$ and 2.58 bits per second. Even though a larger range of $I D$ would strengthen the significance of our results, we had two main reasons to believe that the chosen range would be acceptable. First, previous studies found that children with CP MACS III and IV using a traditional mouse did not follow Fitts's law for IDs above 2 bits (Davies et al 2014). Second, most of our participants with $\mathrm{CP}$ have vision problems and nearly half of them, cognitive disorders. It would be unlikely that they would perform pointing tasks using the same graphical user interfaces that individuals without disabilities. Hence, they would never be exposed to those high values of $I D$ in their daily pointing.

A session with ENLAZA consisted of 4 repetitions of the task with each of the $I D$ values presented in a randomized order. Each participant took part in $4.67 \pm 0.67$ work sessions depending on their availability during the study. In the end, an average value of $M T$ for each of the 4 values of $I D$ was used in the analysis. We define $M T$ as the time the user needs to locate the cursor above the target. A linear regression is computed to fit a model to the data registered for individuals or groups. The data used for the regression of the groups were the global means of $M T$ per $I D$, whereas the regressions for each individual were estimated with the participants means per $I D$. To measure the quality of the fit, the $R^{2}$ or coefficient of determination was calculated from the

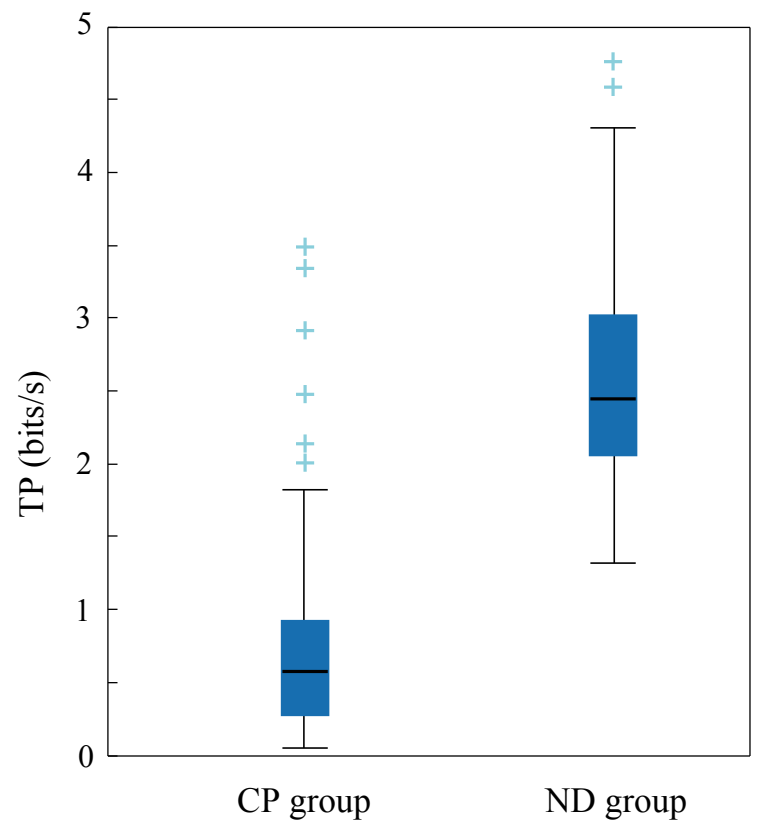

Figure 3: Measure of Throughput (TP) for the two groups. The box plots represent the values measured for each task during the work sessions of cerebral palsy group (CP) and users without motor disabilities (ND). The notches represent $\mathrm{q}_{2} \pm \frac{1.57 \cdot\left(q_{3}-q_{1}\right)}{n}$

residuals of the regression.

\subsection{Assessment of task performance}

Throughput is the metric adopted to analyze task performance. Its value for each user will be estimated following three approaches, TP (as $I D / M T$ ), mean-ofmeans throughput $\left(T P_{\text {avg }}\right)$ and slope-inverse throughput $\left(T P_{i n v}\right)$. Please note that $I D / M T$ is an estimation of the Throughput only in a certain reaching task, $i$.

Table 1: User diagnosis, and topographical and functional capacity classification

\begin{tabular}{lcccc}
\hline User & Diagnosis & Topography & MACS & GMFCS \\
\hline CP1 & D-A & Quadriplegia & 5 & 5 \\
CP2 & D-A & Quadriplegia & 5 & 5 \\
CP3 & Dyskinetic & Quadriplegia & 4 & 4 \\
CP4 & Dyskinetic & Quadriplegia & 5 & 5 \\
CP5 & Spastic & Quadriplegia & 5 & 5 \\
CP6 & Mixed & Diplegia & 5 & 5 \\
\hline
\end{tabular}

* D-A: Dystonic-Athetoid

\section{Results \\ 4.1. Correlation with Fitts's law}

All $M T-I D$ curves as well as the linear model estimated from the regression are plotted in Figs. 4 and 5. Linear regression of the experimental data resulted in 
the following equations (in seconds):

$$
\begin{array}{llll}
C P_{\text {group }}: & M T=-0.825+2.214 \cdot I D, & R^{2}=0.952 \\
N D_{\text {group }}: & M T=+0.474+0.137 \cdot I D, & R^{2}=0.924 \\
C P 1 & : & M T=-2.544+4.253 \cdot I D, & R^{2}=0.839 \\
C P 2 & : & M T=+0.094+1.004 \cdot I D, & R^{2}=0.980 \\
C P 3 & : & M T=+0.068+0.929 \cdot I D, & R^{2}=0.693 \\
C P 4 & : & M T=-2.412+3.939 \cdot I D, & R^{2}=0.784 \\
C P 5 & : & M T=+1.754+3.405 \cdot I D, & R^{2}=0.920 \\
C P 6 & : & M T=+1.387+2.241 \cdot I D, & R^{2}=0.918
\end{array}
$$

Table 3 depicts movement times for each user and $I D$ as well as the value of $R^{2}$. Despite the differences in the task performance, the average motor performance of both groups was proven to fit the linear model proposed by Fitts's law, with $R^{2} 0.924$ (ND) and 0.952 (CP). In terms of individual motor performance, the $R^{2}$ calculated in 3 out of 6 participants with $\mathrm{CP}(\mathrm{CP} 2, \mathrm{CP} 5$, and CP6) was above 0.9. In the case of CP1 (dystonia), it was slightly lower: 0.839 . The lowest values of $R^{2}$ corresponded to $\mathrm{CP} 3$ and $\mathrm{CP} 4$, both of them cases of cerebral palsy with cervical hypotonia.

These results indicate that head controls used by individuals without motor impairments can indeed be modeled by Fitts's law. Similar levels of correlation were found for the group of participants with CP. However, there is poor correlation in individuals with dystonia or hypotonia. This could mean that some profiles of motor disorder found in $\mathrm{CP}$ lead to movements which cannot be predicted by Fitts's law.

\subsection{Task performance}

As expected, non-disabled users (ND) were considerably faster and more efficient than those in the cerebral

Table 3: Movement times and indexes of difficulty and $R^{2}$ of the model.

\begin{tabular}{cccccc}
\hline & \multicolumn{5}{c}{ ID (bits/s) } \\
\cline { 2 - 4 } User & 1.32 & 1.80 & 2.00 & 2.58 & \multirow{2}{*}{$\mathrm{R}^{2}$} \\
\hline CP1 & $3.70 \mathrm{~s}$ & $5.17 \mathrm{~s}$ & $4.58 \mathrm{~s}$ & $9.18 \mathrm{~s}$ & $\mathbf{0 . 8 3 9}$ \\
CP2 & $1.46 \mathrm{~s}$ & $1.92 \mathrm{~s}$ & $1.99 \mathrm{~s}$ & $2.74 \mathrm{~s}$ & $\mathbf{0 . 9 8 0}$ \\
CP3 & $1.52 \mathrm{~s}$ & $1.72 \mathrm{~s}$ & $1.48 \mathrm{~s}$ & $2.72 \mathrm{~s}$ & $\mathbf{0 . 6 9 3}$ \\
CP4 & $3.14 \mathrm{~s}$ & $3.24 \mathrm{~s}$ & $6.62 \mathrm{~s}$ & $7.70 \mathrm{~s}$ & $\mathbf{0 . 7 8 4}$ \\
CP5 & $6.42 \mathrm{~s}$ & $7.22 \mathrm{~s}$ & $9.13 \mathrm{~s}$ & $10.52 \mathrm{~s}$ & $\mathbf{0 . 9 2 0}$ \\
CP6 & $4.51 \mathrm{~s}$ & $5.57 \mathrm{~s}$ & $5.35 \mathrm{~s}$ & $7.41 \mathrm{~s}$ & $\mathbf{0 . 9 1 8}$ \\
\hline CP group & $2.34 \mathrm{~s}$ & $2.98 \mathrm{~s}$ & $3.35 \mathrm{~s}$ & $5.11 \mathrm{~s}$ & $\mathbf{0 . 9 5 2}$ \\
ND group & $660 \mathrm{~ms}$ & $740 \mathrm{~ms}$ & $720 \mathrm{~ms}$ & $840 \mathrm{~ms}$ & $\mathbf{0 . 9 2 4}$ \\
\hline
\end{tabular}

palsy (CP) group during the goal-crossing tasks proposed. The differences can be observed in Fig. 3. The $M T$ registered was smaller (medians in ND and $\mathrm{CP}$ were 0.725 seconds and 3.290 seconds, respectively) and TP significantly larger in the ND group. The median values were $2.510 \mathrm{bits} / \mathrm{s}$ in the ND group and $0.572 \mathrm{bits} / \mathrm{s}$ among the users in the CP group $(\mathrm{p}<0.01)$. Among the participants with $\mathrm{CP}, \mathrm{CP} 3$ scored the best results $\left(T P_{\text {avg }}=1.035 \mathrm{bits} / \mathrm{s}\right)$. Table 4 depicts the results of the tests: the distribution of Movement Time and Throughput measured $(M T, T P)$ as well as the mean-of-means throughput $\left(T P_{a v g}\right)$. It can be observed that $T P_{a v g}$ is a good descriptor of the throughput since it is very similar to $T P$ for all the users.

There was no apparent relation between the performance of the task, described by $T P_{a v g}$, and the goodness of the match with Fitts's law in terms of the calculated $R^{2}$ for none of the groups. This is consistent with the results presented by MacKenzie and Isokoski (2008), who provided evidence that supported the fact the throughput is independent of the speed-accuracy trade-off.

\section{Discussion}

There are studies which test the validity of Fitts's law with users with $\mathrm{CP}$ but they focus on hand control. Severely impaired users (MACS IV and V) cannot use traditional mice and rely on other non-handheld devices. Although most of these devices have been evaluated with Fitts's law, the experiments only included users without motor impairments. Our contribution was to replicate that assessment for the most severely motor impaired using a particular non-handheld device. More precisely, our goal was to evaluate two aspects concerning ENLAZA, a head mouse to access the computer specially developed as an adapted interface for people with severely affected manipulation skills. First, we captured head movements of users without motor disabilities. We were interested in confirming the evidence that suggests that those movements can be modeled by Fitts's law and there is a trade-off between speed and accuracy during the pointing tasks. Secondly, provided that those head movements follow Fitts's law, we wondered if the linear model proposed by Fitts is also valid for users with severe motor and posture disorders such as those found in people with $\mathrm{CP}$ and diplegia or quadriplegia.

In the first phase of the experiments, we measured the movement times of 6 users without motor disabilities and were able to estimate a good regression model of $M T$ as a linear function of the index of difficulty for a series of random goal-crossing tasks. We observed that $M T$ in the ND group (725ms) was not far from the 
Table 4: Distribution of MT and TP and estimation of $\mathrm{TP}_{a v g}$ and $\mathrm{TP}_{i n v}$.

\begin{tabular}{|c|c|c|c|c|c|c|c|c|}
\hline \multirow[b]{2}{*}{ User } & \multicolumn{3}{|c|}{ MT(s) } & \multicolumn{3}{|c|}{$\mathrm{TP}($ bits/s) } & \multirow[b]{2}{*}{$\mathrm{TP}_{a v g}$} & \multirow[b]{2}{*}{$\mathrm{TP}_{i n v}$} \\
\hline & $\mathrm{q}_{1}$ & $\mathrm{q}_{2}$ & $\mathrm{q}_{3}$ & $\mathrm{q}_{1}$ & $\mathrm{q}_{2}$ & $\mathrm{q}_{3}$ & & \\
\hline CP1 & 2.968 & 5.134 & 9.003 & 0.197 & 0.363 & 0.577 & 0.343 & 0.235 \\
\hline $\mathrm{CP} 2$ & 1.331 & 1.931 & 3.000 & 0.640 & 0.944 & 1.405 & 0.953 & 0.996 \\
\hline CP3 & 1.284 & 1.727 & 3.611 & 0.532 & 1.033 & 1.560 & 1.035 & 1.076 \\
\hline $\mathrm{CP} 4$ & 2.545 & 4.862 & 8.565 & 0.241 & 0.383 & 0.678 & 0.371 & 0.253 \\
\hline CP5 & 4.609 & 8.301 & 13.20 & 0.121 & 0.234 & 0.439 & 0.231 & 0.293 \\
\hline CP6 & 3.050 & 5.769 & 9.417 & 0.193 & 0.339 & 0.634 & 0.337 & 0.446 \\
\hline $\mathrm{CP}_{\text {group }}$ & 1.801 & 3.290 & 7.022 & 0.278 & 0.572 & 1.004 & 0.559 & 0.451 \\
\hline $\mathrm{ND}_{\text {group }}$ & 0.632 & 0.725 & 0.861 & 2.065 & 2.510 & 3.081 & 2.608 & 7.299 \\
\hline
\end{tabular}
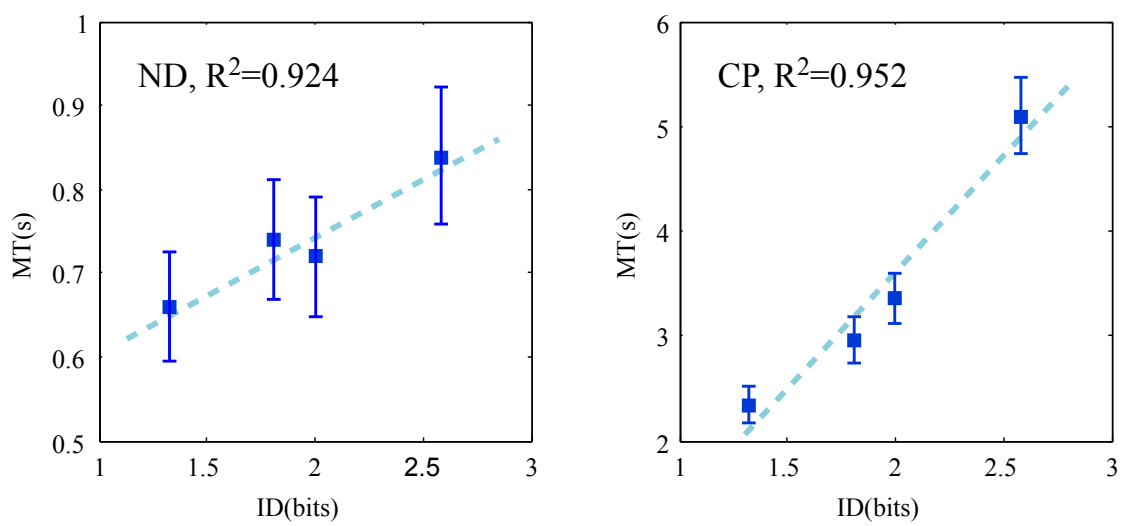

Figure 4: Representation of $M T-I D$ curves measured in the experiments with the interface ENLAZA for both study groups: non-disabled users (ND) and users with cerebral palsy (CP). The value of $R^{2}$ is an indicator of the good correlation with Fitts's law.

values measured with standard mice by Luo and Vogel (2014), 716ms, or Apitz et al. (2010), 500-600ms, for a stylus. The second phase was also satisfactory, as we achieved to estimate linear models for 6 users with CP, even though they performed considerably poorer than the users without disabilities using the device. Davies et al. (2014) observed similar differences between typically developing youths (TDY) and youths with CP, MACS III and IV. Interestingly, they didn't find significant differences for IDs above 2 bits as we did. They concluded that Fitts's law is not valid for users who are MACS III and IV.

\subsection{The importance of the transfer function}

Our experiment and the one conducted by Davies et al. (2014) differ in several aspects (pointing device, pointing strategy, task, etc). One of them is the transfer function or control-display gain relationship that the devices used. Whereas Davies used the Windows default mode or enhance pointer precision (thoroughly de- scribed by Casiez and Roussel (2011)), ENLAZA integrates a RKF instead. The RKF filters some of the involuntary movements and reduces the number of submovements. It may be the key to explaining why we found a strong correlation between $M T$ and $I D$. In future studies, it would be interesting to evaluate the effects of RKF on the validity of Fitts's law for individuals with $\mathrm{CP}$ that are MACS I-III using a mouse.

\subsection{The use of goal-crossing instead of bi-directional tapping and selection}

Head mice such as ENLAZA enable cursor control with head movements but selection (e.g. click actions) requires the use of extra techniques such as dwell time. Bi-directional tapping and selection with ENLAZA would need the use of dwell time. Although some of our participants in ASPACE are indeed very experienced with the dwell time to select elements on the screen, others have difficulties staying on a target the required time. In their study, Raya et al. (2012) 
assessed ENLAZA with bi-directional tapping and selection. They could only recruit 3 users with $\mathrm{CP}$ and measured poorer correlations. In this study, we use the goal-crossing to be able to recruit a larger number of participants.

While we could have combined goal-crossing and bidirectional tapping and asked our participants to perform "bi-directional goal-crossing", we and our partners in ASPACE-Cantabria believe that motivational aspects are important for the success of the experiments. While we risked measuring poorer correlations with Fitts's law (although the studies that we reviewed suggest that Fitts's law would still be followed), we minimized the number of drop outs with an exercise that the participants found more engaging than the traditional bi-directional tapping.

\subsection{Explaining the impact of posture and motor control in goal-crossing tasks}

Three out of the six members of the group with $\mathrm{CP}$ showed good correlation with Fitts's law (values of $R^{2}$ greater than 0.9 ). We believe that low $R^{2}$ may be a consequence of a poorer postural control since the worst values of correlation were measured for users with decremented cervical tone and dyskinetic CP. Almanji et al. (2014) suggested that the movements of individuals with CP cannot be modeled by Fitts's law because movement instability and pauses are common in their pointing tasks. Gump et al. (2002) theorized that "unreliable visual feedback" could be the source of errors and ballistic movements in users with moderate to severe CP. ENLAZA's RKF should minimize the effects of these anomalies by preventing most involuntary movements from affecting the trajectory of the cursor as Raya et al. (2012) and Velasco et al. (2016) proved. Nonetheless, the slightly lower value of $R^{2}$ achieved by CP1 could be due to the residuals of his involuntary movements. We consider that more specific tests should be run with participants with hypotonic CP and or involuntary movements to establish whether their head movements follow Fitts's law. It would also be interesting to analyze different trajectory directions. That could give us some insight into how muscle weakness is affecting head movements and the interaction with ENLAZA.

\subsection{Experimental concerns and further work}

There are some limitations that are inherent to the population under study and the experiment itself. To begin with, the disability of the sample in our $\mathrm{CP}$ group (as CP itself) is rather heterogeneous in terms of muscular tone, postural control, involuntary movement and intellectual ability. Six participants with $\mathrm{CP}$ (one of them was MACS VI; the rest, MACS V) is indeed a small number for any study, but the fact is that these limitations are shared with most of the publications reviewed and that points out the difficulties researchers find to recruit a large population of volunteers with $\mathrm{CP}$ and similar levels of severity. For instance, Davies et al. (2010b) recruited 6 users MACS IV and 8 MACS V. Davies et al. (2014) and Almanji et al. (2014) recruited 9 users MACS III and 3 MACS IV, but none MACS V; Saavedra et al. (2010), 3 users with CP GMFCS III. In addition, we had to deal with the daily routines of users with CP in ASPACE-Cantabria (with tightly scheduled occupational and physical therapy, lectures, transportation, lunch times, etc), which do not allow systematic testing. Instead, we were forced to plan shorter work sessions during a wider period of time that could be "squeezed" into their timetables. Finally, some aspects such as motivation or fatigue were not quantified although they may play an important role in the performance of the task. However, we found a consensus with the therapists in ASPACE-Cantabria and approached the subject by designing a protocol based on videogames in order to enhance the user's attention and minimize the effects of tedium that a traditional Fitts's law study could cause in our users over the weeks of experimentation.

The participants with $\mathrm{CP}$ and the therapist expressed their satisfaction with the performance of the ENLAZA. Although they found it "easy to use", some individuals showed signs of fatigue after a few minutes during their first experiences with the device. This could be due to muscle weakness, as no signs of fatigue were found during the experiments. Nevertheless, we will include the assessment of effort in future studies to complete a validation of the usability. To gather a larger group of participants with CP to take part in a series of systematic tests based on the traditional bi-directional tapping paradigm would be desirable for more robust statistical significance. But this goal can only be achieved if we succeed to integrate this kind of exercises into these users' daily routines.

\subsection{Application of new input algorithms}

The strategy of interaction with ENLAZA, absolute control, is based on head posture and defines a direct relation between head orientation and cursor movement. The results of these experiments suggest that this strategy together with the RKF are a usable solution for most users with CP. However, we could implement new modes of control based on velocity rather than orientation, as the one proposed by Rudigkeit et al. (2015). In 
parallel to Rudigkeit, we have developed a relative control based on the angular velocity measured by the gyroscopes. Preliminary results show that even if the user is leaning forward or backward due to muscle weakness, he or she will still be able to move the mouse pointer by small head movements. It is possible that those low values of $R^{2}$ estimated in this study could be improved if those participants were using the relative control instead of the absolute control. In future studies, the relative control will be tested in users with cervical hypotonia. The aim of these studies will be to compare the task performance of the two possible control modes and to assess the correlation with Fitts's law.

\section{Conclusion}

This work contributed to the evaluation of Fitts's law on individuals with and without $\mathrm{CP}$ who used a head mouse named ENLAZA. The participants with CP were MACS IV-V and could not access the computer with traditional pointing devices such as mice.

As expected, the movements of users without motor impairments followed Fitts's law and we found a good correlation for at least three users with CP (dystonic, spastic and mixed). We also found a weaker correlation for a participant with dystonic CP and involuntary movements. Two users with dyskinetic CP and decremented muscular tone showed no correlation with Fitts's law. These results encourage us to continue researching and developing new interaction techniques and facilitation algorithms towards the design of a universal interface for individuals with $\mathrm{CP}$ and other motion-impaired users.

\section{Acknowledgments}

Authors would like to thank the members and staff in ASPACE Cantabria, specially Teresa González and Antonio Ruiz. This work was possible thank to ABC EU Project, the InterAAC Project (RTC-20154327-1), CP-Walker Project (DPI2012-39133-C03-01), INTERPLAY Project (RTC-2014-1812-1) and IVANPACE Project, which was funded by Fundación Caja Cantabria. A. Clemotte would also like to thank to Itaipu Binacional for its support.

\section{References}

Accot, J., Zhai, S., 2002. More than dotting the i's - Foundations for crossing-based interfaces. Proceedings of the SIGCHI conference on Human factors in computing systems CHI '02 (4), 73-80.
Almanji, A., Davies, T. C., Stott, N. S., 2014. Using cursor measures to investigate the effects of impairment severity on cursor control for youths with cerebral palsy. International Journal of $\mathrm{Hu}-$ man Computer Studies 72 (3), 349-357.

Alonso, R., Causse, M., Vachon, F., Parise, R., Dehais, F., Terrier, P., 2013. Evaluation of head-free eye tracking as an input device for air traffic control. Ergonomics 56 (2), 246-55.

Apitz, G., Guimbretière, F., 2004. CrossY: A Crossing-Based Drawing Application. Proceedings of the 17th annual ACM symposium on User interface software and technology - UIST '04 6 (2), 3.

Apitz, G., Guimbretière, F., Zhai, S., 2010. Foundations for designing and evaluating user interfaces based on the crossing paradigm. ACM Transactions on Computer-Human Interaction 17 (2), 1-42.

Balakrishnan, R., 2004. "Beating” Fitts' law: virtual enhancements for pointing facilitation. International Journal of Human-Computer Studies 61 (6), 857-874.

Bax, M., Goldstein, M., Rosenbaum, P., Leviton, A., Paneth, N., Dan, B., Jacobsson, B., Damiano, D., Jul. 2005. Proposed definition and classification of cerebral palsy, April 2005. Developmental Medicine \& Child Neurology 47 (8), 571-576.

Bible, J. E., Biswas, D., Miller, C. P., Whang, P. G., Grauer, J. N., 2010. Normal functional range of motion of the cervical spine during 15 activities of daily living. Journal of spinal disorders \& techniques 23 (1), 15-21.

Biswas, P., Langdon, P., 2013. A new interaction technique involving eye gaze tracker and scanning system. Proceedings of the 2013 Conference on Eye Tracking South Africa - ETSA '13, 67-70.

Cans, C., feb 2007. Surveillance of cerebral palsy in Europe: a collaboration of cerebral palsy surveys and registers. Developmental Medicine \& Child Neurology 42 (12), 816-824.

Card, S. K., English, W. K., Burr, B. J., 1978. Evaluation of mouse, rare-controlled isometric joystick, step keys, and text keys for text selection on a CRT. Ergonomics 8, 601-613.

Casiez, G., Roussel, N., 2011. No more bricolage!: methods and tools to characterize, replicate and compare pointing transfer functions. Proceeding UIST ' 11 Proceedings of the 24th annual ACM symposium on User interface software and technology, 603-614.

Chen, X., Wang, Z. J., 2013. Pattern recognition of number gestures based on a wireless surface EMG system. Biomedical Signal Processing and Control 8 (2), 184-192.

Davies, T., Mudge, S., Ameratunga, S., Stott, N., 2010a. Enabling self-directed computer use for individuals with cerebral palsy: a systematic review of assistive devices and technologies. Developmental Medicine \& Child Neurology 52 (6), 510-516.

Davies, T. C., AlManji, A., Stott, N. S., 2014. A cross-sectional study examining computer task completion by adolescents with cerebral palsy across the Manual Ability Classification System levels. Developmental Medicine \& Child Neurology 56 (12), 1180-1186.

Davies, T. C., Chau, T., Fehlings, D. L., Ameratunga, S., Stott, N. S., 2010b. Youth With Cerebral Palsy With Differing Upper Limb Abilities: How Do They Access Computers? Archives of Physical Medicine and Rehabilitation 91 (12), 1952-1956.

Eliasson, A.-C., Krumlinde-Sundholm, L., Rösblad, B., Beckung, E., Arner, M., Ohrvall, A.-M., Rosenbaum, P., jul 2006. The Manual Ability Classification System (MACS) for children with cerebral palsy: scale development and evidence of validity and reliability. Developmental Medicine \& Child Neurology 48 (7), 549-54.

Felton, E. A., Williams, J. C., Vanderheiden, G. C., Radwin, R. G., 2012. Mental workload during brain-computer interface training. Ergonomics 55 (5), 526-537.

Fitts, P. M., Jun. 1954. The information capacity of the human motor system in controlling the amplitude of movement. Journal of experimental psychology. General 47 (6), 381-91.

Gump, A., LeGare, M., Hunt, D. L., jun 2002. Application of Fitts' Law to Individuals with Cerebral Palsy. Perceptual and Motor 
Skills 94 (3), 883-895.

Hurst, A., Hudson, S. E., Mankoff, J., Trewin, S., Box, P. O., Ny, Y. H., 2008. Automatically Detecting Pointing Performance. Proceedings of the 13th international conference on Intelligent user interfaces, 11-19.

Jagacinski, R. J., Monk, D. L., 1985. Fitts' Law in two dimensions with hand and head movements. Journal of motor behavior 17 (1), 77-95.

Johnson, A., Sep. 2002. Prevalence and characteristics of children with cerebral palsy in Europe. Developmental medicine and child neurology 44 (9), 633-40.

Kamavuako, E. N., Scheme, E. J., Englehart, K. B., 2014. On the usability of intramuscular EMG for prosthetic control: A Fitts' Law approach. Journal of electromyography and kinesiology : official journal of the International Society of Electrophysiological Kinesiology 24 (5), 770-7.

Kim, M., Chae, Y., Jo, S., 2013. Low-Cost Hybrid Interface with EEG and Eye Movement for Multi-Directional Target Selection, 763766

Kim, M., Kim, B. H., Jo, S., 2015. Quantitative evaluation of a lowcost noninvasive hybrid interface based on EEG and eye movement. IEEE transactions on neural systems and rehabilitation engineering : a publication of the IEEE Engineering in Medicine and Biology Society 23 (2), 159-68.

Lin, Y.-L., Chen, M.-C., Yeh, C.-C., Yeh, Y.-M., Wang, H.-P., 2008. Assisting an adolescent with cerebral palsy to entry text by using the chorded keyboard. In: Miesenberger, K., Klaus, J., Zagler, W., Karshmer, A. (Eds.), Computers Helping People with Special Needs. Vol. 5105 of Lecture Notes in Computer Science. Springer Berlin Heidelberg, pp. 1177-1183.

Luo, Y., Vogel, D., 2014. Crossing-based Selection with Direct Touch Input. Proceedings of the SIGCHI Conference on Human Factors in Computing Systems, 2627-2636.

MacKenzie, I. S., Isokoski, P., 2008. Fitts' throughput and the speedaccuracy tradeoff. Proceeding of the twenty-sixth annual CHI conference on Human factors in computing systems - CHI '08, 1633.

Olds, K. C., Sibenaller, S., Cooper, R. A., Ding, D., Riviere, C., 2008. Target prediction for icon clicking by athetoid persons. 2008 IEEE International Conference on Robotics and Automation (2), 2043 2048

Quinn, P., Cockburn, A., Delamarche, J., 2013. Examining the costs of multiple trajectory pointing techniques. International Journal of Human Computer Studies 71 (4), 492-509.

Radwin, R. G., Vanderheiden, G. C., Lin, M. L., Aug. 1990. A method for evaluating head-controlled computer input devices using Fitts' law. Human factors 32 (4), 423-438.

Raya, R., Rocon, E., Ceres, R., Belda, J. M., Laparra, J., Feb. 2013 Positive and negative motor signs of head motion in cerebral palsy an analysis based on a wearable inertial human computer interface. In: 2013 ISSNIP Biosignals and Biorobotics Conference: Biosignals and Robotics for Better and Safer Living (BRC). Ieee, pp. 1-5.

Raya, R., Rocon, E., Gallego, J. A., Ceres, R., Pons, J. L., Jan. 2012. A robust Kalman algorithm to facilitate human-computer interaction for people with cerebral palsy, using a new interface based on inertial sensors. Sensors (Basel, Switzerland) 12 (3), 3049-67.

Rempel, D., Camilleri, M. J., Lee, D. L., 2014. The design of hand gestures for human-computer interaction: Lessons from sign language interpreters. International Journal of Human Computer Studies 72 (10-11), 728-735.

Rudigkeit, N., Gebhard, M., Graser, A., may 2015. Evaluation of control modes for head motion-based control with motion sensors. In: 2015 IEEE International Symposium on Medical Measurements and Applications (MeMeA) Proceedings. IEEE, pp. 135-140.

Saavedra, S., Woollacott, M., Van Donkelaar, P., 2010. Head stability during quiet sitting in children with cerebral palsy: Effect of vision and trunk support. Experimental Brain Research 201 (1), 13-23.

Saavedra, S. L., Woollacott, M. H., 2015. Segmental Contributions to Trunk Control in Children With Moderate-to-Severe Cerebral Palsy. Archives of Physical Medicine and Rehabilitation.

Scheme, E., Lock, B., Hargrove, L., Hill, W., Kuraganti, U., Englehart, K., 2013. Motion Normalized Proportional Control for Improved Pattern Recognition Based Myoelectric Control. IEEE Transactions on Neural Systems and Rehabilitation Engineering 22 (1), 149-157.

Scheme, E. J., Englehart, K. B., 2013. Validation of a selective ensemble-based classification scheme for myoelectric control using a three-dimensional Fitts' Law test. IEEE Transactions on Neural Systems and Rehabilitation Engineering 21 (4), 616-623.

Schlögl, A., Vidaurre, C., Müller, K.-R., 2009. Adaptive methods in BCI research - an introductory tutorial, 1-28.

Simpson, R., Koester, H., LoPresti, E., 2006. Evaluation of an adaptive row/column scanning system. Technology and disability 18 (3), 127-38

Soukoreff, R. W., Mackenzie, I. S., Dec. 2004. Towards a standard for pointing device evaluation, perspectives on 27 years of Fitts law research in HCI. International Journal of Human-Computer Studies 61 (6), 751-789.

Špakov, O., Isokoski, P., Majaranta, P., 2014. Look and Lean : Accurate Head-Assisted Eye Pointing. Proceedings of the ETRA conference 1 (212), 35-42.

Surakka, V., Illi, M., Isokoski, P., 2004. Gazing and frowning as a new human-computer interaction technique. ACM Transactions on Applied Perception (TAP) 1 (1), 40-56.

Tuisku, O., Rantanen, V., Pakov, O., Surakka, V., Lekkala, J., 2014. Pointing and Selecting with Facial Activity. Interacting with Computers.

Tuisku, O., Surakka, V., Vanhala, T., Rantanen, V., Lekkala, J., 2012. Wireless Face Interface: Using voluntary gaze direction and facial muscle activations for human-computer interaction. Interacting with Computers 24 (1), 1-9.

Van Naarden Braun, K., Doernberg, N., Schieve, L., Christensen, D., Goodman, A., Yeargin-Allsopp, M., jan 2016. Birth Prevalence of Cerebral Palsy: A Population-Based Study. Pediatrics 137 (1), e20152872.

Velasco, M. A., Raya, R., Ceres, R., Clemotte, A., Ruiz Bedia, A., Gonzalez Franco, T., Rocon, E., sep 2016. Positive and Negative Motor Signs of Head Motion in Cerebral Palsy: Assessment of Impairment and Task Performance. IEEE Systems Journal 10 (3), 967-973.

Wall, S., Harwin, W., 2000. Quantification of the effects of haptic feedback during a motor skills task in a simulated environment. Proceedings of second PHANToM users research symposium.

Williams, M. R., Kirsch, R. F., 2015. Evaluation of head orientation and neck muscle EMG signals as command inputs to a humancomputer interface for individuals with high tetraplegia. Journal of neuroengineering and rehabilitation 12 (25).

Wobbrock, J. O., Jansen, A., Shinohara, K., 2011. Modeling and predicting pointing errors in two dimensions. In: Proceedings of the 2011 annual conference on Human factors in computing systems CHI '11. ACM Press, New York, New York, USA, p. 1653.

Wu, T.-F., Chen, M.-C., 2007. Performance of different pointing devices on children with cerebral palsy. In: Stephanidis, C. (Ed.), Universal Access in Human-Computer Interaction. Applications and Services. Vol. 4556 of Lecture Notes in Computer Science. Springer Berlin Heidelberg, pp. 462-469.

Zhao, J., Soukoreff, R. W., Ren, X., Balakrishnan, R., 2014. A model of scrolling on touch-sensitive displays. International Journal of Human-Computer Studies 72 (12), 805-821. 


\section{Vitae}

Miguel A. Velasco received the Telecommunication Engineering degree from Universidad de Zaragoza, Spain, in 2009 and the Master's degree in Biomedical Engineering, with specialization in bioelectronics and biomedical instrumentation, from Universitat Politècnica de València, Spain, in 2012. In 2012, he was with the Interuniversity Institute of Bioengineering Research and Technology Oriented to the Human Being (i3BH), Valencia, as a Research Associate focused on biosignal acquisition and processing. He is currently working towards his Ph.D. degree at the Neural and Cognitive Engineering group of the Spanish Research Council (CSIC), working on alternative computer access and rehabilitation systems for people with motor disorders.

Alejandro Clemotte was born in Paraguay. He graduated with honors in the Catholic University of Asunción, in 2011. He is Master in Engineering of Electronic Systems by the Polytechnic University of Madrid and graduated with honors in 2013, with a study about the ability of gaze tracking system when it is used by people with cerebral palsy. He is currently working on his doctoral thesis with the Neural and Cognitive Engineering group of the Spanish Research Council (CSIC). He studies alternative computer access tools for people with cerebral palsy, with special emphasis on gaze tracking systems and its impact in the cognitive level of the users and metrics and models for a better description of these users.

Rafael Raya received the Electronic and Automatic Engineering degree from the University of Córdoba, Spain, in 2006 and the M.S. and Ph.D. degrees from the University of Alcalá, Madrid, Spain, in 2008 and 2011, respectively. He was a Postdoctoral Fellow with the Harvard Medical School, Boston, USA, and with MOVE Institute (Vrije Amsterdam Universiteit, Amsterdam, The Netherlands). He is the author or coauthor of more than 40 publications, including international journals and conferences, and is a Reviewer of several international journals. He has actively participated in a number of national and international research and technological development projects. His research activity is focused on assistive devices for people with cerebral palsy. Dr. Raya is currently a Technical Coordinator with the Iberoamerican Association for Assistive Technology (AITADIS). He received the 2011 Best Spanish Ph.D. Thesis in Robotics from the Spanish
Committee for Automation and the TR35 Award from Massachusetts Institute of Technology's journal, Technology Review (2013).

Dr Ramón Ceres, is Professor of Research at the Bioengineering Group of the Spanish National Council for Science Research (CSIC), received his Ph.D. at the Universidad Complutense of Madrid. He has leaded numerous $\mathrm{R} \& \mathrm{D}$ national and international projects, contributing to the creation of two spin off and other transfer technology processes. Is author of more than 250 publications centred on the field of research of the Assistive Technologies, particularly on sensors, interfaces and signal processing. He has worked in different positions, mainly in the rehabilitation field, being Founder President of Latin-American Association for Assistive Technologies AITADIS.

Eduardo Rocon was born in Vitoria, Brazil (1979). He graduated in Electrical Engineering at Universidade Federal do Espiríto Santo (UFES) in 2001. From 1999 through 2000 he worked as a research associate at Laboratório de Automação Inteligente and successfully held a CNPq scholarship at UFES. Subsequently he moved to Spain to pursue a Ph.D. degree in Industrial Engineering at Universidad Politécnica de Madrid with Prof. Barrientos and Prof. Pons. His Ph.D. thesis (2006), for which he was awarded the Georges Giralt PhD Award (2008), focused on the development of a rehabilitation robotic exoskeleton that provides a means of testing and validating non grounded control strategies for robotic exoskeletons for active upper limb tremor suppression. Dr. Rocon continued his work in tremor suppression and the application of neuroprosthetics and neurorobotics in rehabilitation on a post-doctoral contract from 2006 to 2009. In 2009, Dr. Rocon was awarded with the prestigious Ramón y Cajal contract to continue developing his activities. At the age of 30 , Dr. Rocon got a tenured researcher position (2010-present) at the $\mathrm{gNeC}$ at CSIC. His career has recently been awarded the prestigious Juan Lopez de Peñalver Award of the Spanish Royal Academy of Engineering. Dr. Rocon's multidisciplinary work has contributed to different aspects of robotics, neuroscience and medicine. His research activities have generated more than 40 publications in indexed journals, 1 book, 9 book chapters, more than 50 articles in international conferences, 50 articles in national conferences, 5 articles in journal of scientific diffusion, and 7 patents. 

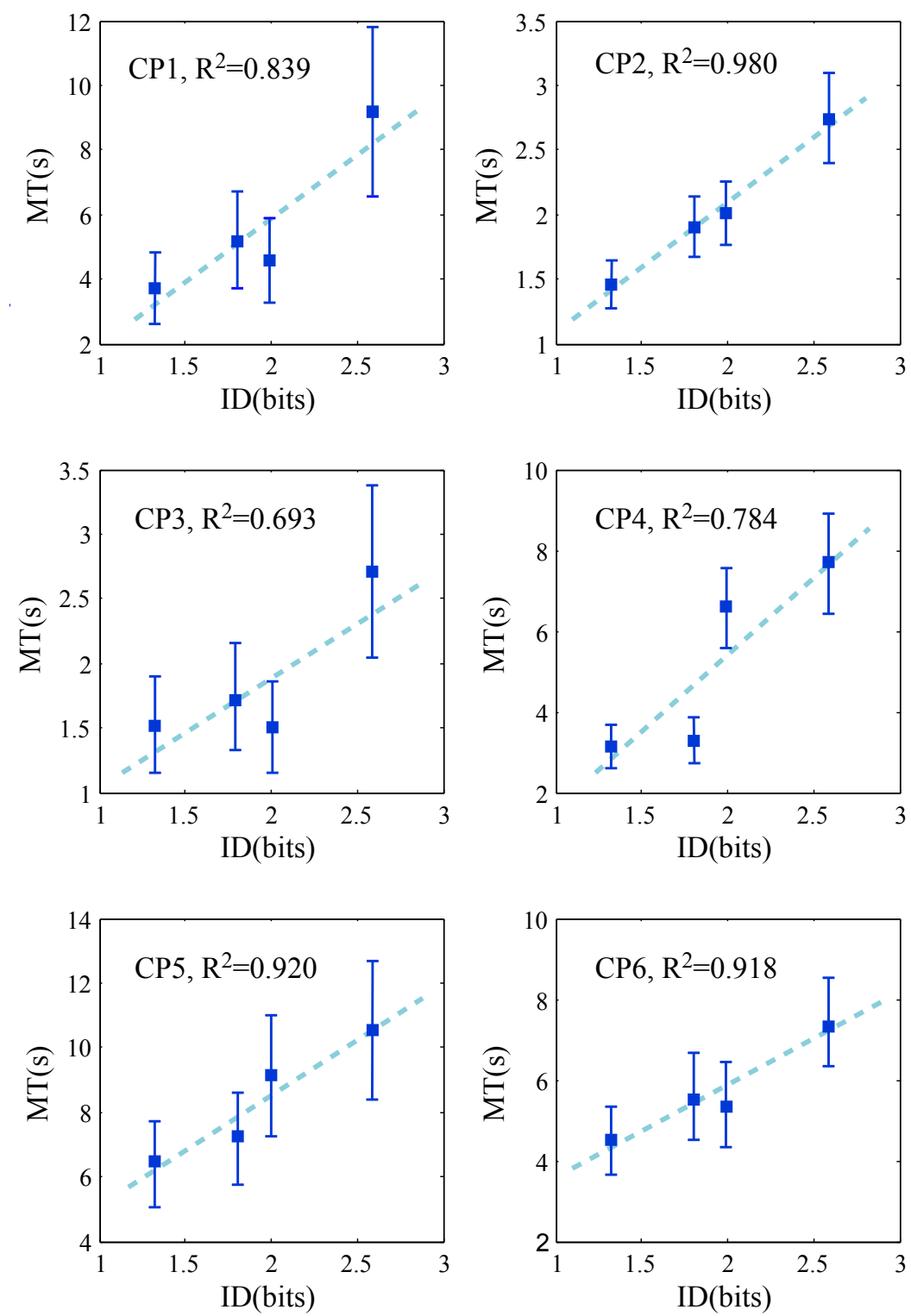

Figure 5: Representation of MT-ID curves measured in the experiments with the interface ENLAZA for the CP group. The value of $R^{2}$ is an indicator of the good correlation with Fitts's law in 4 out of 6 users. 